\title{
A rare case of confusion
}

\author{
Author: Tun Zan Maung ${ }^{\mathrm{A}}$
}

\section{Case presentation}

An 87-year-old woman, previously fully independent, was admitted to the oncology ward with a myriad of symptoms: bilateral hearing impairment, dizziness, unsteadiness, inability to walk, weakness and confusion for 2 weeks. She had a history of urothelial bladder cancer with predominantly liver and nodal involvement. She had transurethral resection of a bladder tumour in 2016 and completed adjuvant radiotherapy and chemotherapy, which comprised standard gemcitabine and carboplatin. Unfortunately, her cancer progressed and she was started on pembrolizumab in April 2018; mid-scan she showed good response to treatment by RECIST criteria.

Clinical examination revealed slightly reduced power in her lower limbs and bilateral hearing impairment. Blood test results were unremarkable except for mild hyponatraemia (130 mmol/L). Inflammatory markers were not high. Measurements of serum glucose, thyroid function test, hydroxycobalamin, folate, calcium, cortisol and arterial blood gas were normal. Computed tomography (CT) and magnetic resonance imaging (MRI) of her head did not show any significant pathology. A neurologist gave a differential diagnosis of metastasis to skull base and hearing apparatus, viral or immunotherapy-related encephalitis and non-convulsive status epilepticus. Intravenous acyclovir and methylprednisolone (5 days) were started empirically.

On further neurological tests, electroencephalography (EEG) showed mild encephalopathy but no features of epileptic activity or encephalitis, and lumbar puncture showed high cerebrospinal fluid (CSF) protein $(1.6 \mathrm{~g} / \mathrm{L})$ and low glucose $(0.7 \mathrm{mmol} / \mathrm{L})$. In culture, no organism was grown. Extended viral PCR and an autoimmune encephalitis screen were negative. Unfortunately, cytology showed atypical cells consistent with malignant meningitis. Immunohistochemistry revealed that both cytokeratin (CK) $\mathrm{AE} 1 / 3$ and $\mathrm{CK} 7$ were positive. CK20 and S100 were negative. Tumour cells were consistent with metastatic carcinoma cells and compatible with cells from her known bladder cancer.

After a lengthy discussion with the patient's family, we adopted a best supportive care approach as the prognosis is guarded, especially with involvement of the central nervous system. This case illustrates that diagnosis of carcinomatous meningitis can be difficult, as the heterogeneous nature of presentation often delays the diagnosis. ${ }^{1,2}$

\section{Conflicts of interest}

None declared.

\section{References}

1 Umezawa Y, Shirotake S, Kaneko G et al. Meningeal carcinomatosis from bladder cancer: a case report and review of the literature. Mol Clin Oncol 2019;10:506-10.

2 Foo CT, Burrell LM, Johnson DF. An unusual presentation of carcinomatous meningitis. Oxf Med Case Reports 2016;8:omw068. 